\title{
Ultra-High Resolution Ca II Observations of the LISM
}

\author{
Barry Y. Welsh ${ }^{1}$, Rosine Lallement ${ }^{2}$, and Ian Crawford ${ }^{3}$ \\ 1 Eureka Scientific, Oakland, CA 94602, USA \\ 2 Service d'Aeronomie du CNRS, Verrieres le Buisson, France \\ 3 University College London, London WC1E 6BT, England
}

\begin{abstract}
We report on the status of a long-term program of mapping the velocity structure of the local ISM using ultra-high resolution observations of CaII K-line absorption towards nearby $\mathrm{B}$ and A-type stars. Absorption components common to the local interstellar cloud (LIC) have been detected in many (but not all) linesof-sight. Our preliminary results indicate that the local ISM has a complex velocity pattern of inhomogeneous absorption, indicative of a shocked filamentary nature.
\end{abstract}

\section{Introduction}

The Ultra-High Resolution Facility (UHRF) at the Anglo-Australian $3.9 \mathrm{~m}$ Telescope is an echelle spectrograph capable of a resolving power of $\mathrm{R}=\lambda$ / $\delta \lambda=1,000,000\left(0.3 \mathrm{~km} \mathrm{~s}^{-1}\right)$, and is described in detail in Diego et al (1995). Such high spectral resolution enables discrete, but closely spaced, absorption components to be not only resolved, but also located with a precise velocity $\left(0.1 \mathrm{~km} \mathrm{~s}^{-1}\right)$. This instrument is thus ideal for absorption studies of the diffuse gas "cloudlets" known to lie within 40pc of the Sun, in which previous lower resolution studies by Lallement et al (1986) and Vallerga et al (1993) have shown a complex pattern of absorption components even for lines-ofsight towards stars within 5pc. Using a Doppler triangulation method on the observed projected velocities of absorption components detected towards nearby stars, Lallement and Bertin (1992) identified two common absorption features (separated by only a few $\mathrm{km} \mathrm{s}^{-1}$ ) due to the local interstellar cloud (LIC) and the local "G" cloud. Both of these very nearby cloudlets may well completely surround the Sun, and hence should be detected in line-of-sight absorption studies towards all stars.

The temperature, $T_{c}$, of such a diffuse gas cloudlet can be directly determined from the Doppler broadening parameter (b-value) of the observed absorption lines, if turbulence has a small effect. For the very nearest of these clouds (the LIC) ultraviolet observations indicate the rms turbulent velocity to be $<1 \mathrm{~km} \mathrm{~s}^{-1}$ (Linsky and Wood 1995), and hence for an assumed LIC kinetic temperature of $7000 \mathrm{~K}$, the CaII K-line Doppler broadening parameter is $2.4 \mathrm{~km} \mathrm{~s}^{-1}$. This observed line width is far greater than the intrinsic UHRF instrumental width, and hence we are able to gain an unambiguous direct measurement of the temperature of clouds in the local ISM. 
In this paper we report on the present status of our long-term program of mapping the velocity structure of the local ISM using UHRF spectroscopy of the CaII K-line at $3333 \AA$ observed in absorption towards nearby $\mathrm{B}$ and A-type stars. These observations, when used in conjunction with HST GHRS and STIS ultraviolet data, can provide better insights into (a) the physical distribution and number of diffuse interstellar clouds in the local ISM, (b) the ionization state and balance of the local ISM, (c) the density, temperature and pressure of local gas clouds, and (d) the kinematics of the local gas clouds.

\section{Observations}

The galactic distribution of CaII absorption column density, N(CaII), in lineof-sight towards 30 stars within 50 pc has been presented by Vallerga et al (1993). For most lines of sight, the level of CaII absorption is weak (i.e. $\mathrm{N}$ (CaII) $<11.0 \mathrm{~cm}^{-2}$ ). However, 2 of the 3 lines-of-sight with an appreciably high level of CaII absorption all lie in the general direction of Ophiuchus at a distance of $\sim 20 \mathrm{pc}$. Our recent observations of $51 \mathrm{Oph}$ also indicate high levels of CaII absorption in this direction (Crawford, Craig and Welsh 1997). The local distribution of neutral sodium column density, $\mathrm{N}(\mathrm{NaI})$, follows a similar pattern in that there is a distinct paucity of cold, neutral gas within $50 \mathrm{pc}$ (Welsh et al 1994). Bertin et al (1993) have noted that the average value of $\mathrm{N}(\mathrm{NaI}) / \mathrm{N}(\mathrm{CaII})$ is $<0.2$ for lines-of-sight within $30 \mathrm{pc}$, indicative of the presence of warm $(7000 \mathrm{~K})$ LISM gas in which Ca is depleted by a factor of 40.

UHRF CaII spectra have already been presented for local lines-of-sight (d $<40 \mathrm{pc}$ ) towards $\alpha$ Oph, $\alpha$ Gru and $\alpha$ Eri (Crawford and Dunkin 1995), $\alpha$ Cen (Crawford 1994), and $\gamma \mathrm{Aqr}, \iota$ Cen, $\epsilon$ Gru, $\alpha$ Hyi, $\gamma$ Oph and 51 Oph (Crawford, Craig and Welsh 1997). Absorption components due to the LIC and/or the closely associated "G Cloud" have been identified in most lines-ofsight, but only in the case of the star $51 \mathrm{Oph}$ are both clouds simultaneously detected. In addition, the lines-of-sight to $\gamma \mathrm{Oph}$ and $\alpha$ Eri reveal two and four absorption components respectively, but none of these component velocities can be associated with either the LIC or "G Cloud". Furthermore, the nearest star to the Sun, $\alpha$ Cen, has no detectable CaII absorption to the low level of $\mathrm{N}(\mathrm{CaII})<5 \times 10^{9} \mathrm{~cm}^{-2}$.

Most of the CaII clouds detected in the LISM possess velocity dispersions of $\mathrm{b}$ value $\sim 2 \mathrm{~km} \mathrm{~s}^{-1}$, suggesting physical conditions that are similar to those found for the LIC (i.e. $\mathrm{T} \sim 7000 \mathrm{~K}, \mathrm{n} \sim 0.1 \mathrm{~cm}^{-3}$ ). However, several anomalous clouds have been found which do not fit into this category. For example, the cloud detected towards $51 \mathrm{Oph}$ at a velocity of $-20.3 \mathrm{~km} \mathrm{~s}^{-1}$ has an observed $b$ value of only $0.6 \mathrm{~km} \mathrm{~s}^{-1}$ which corresponds to a cloud temperature of $<870 \mathrm{~K}$. Also, the single cloud observed towards $\gamma \mathrm{Aqr}$ ( $\mathrm{d}$ $=23 \mathrm{pc}$ ) at a velocity corresponding the LIC has an inferred temperature of only $3500 \mathrm{~K}$ (assuming low turbulence). 
In figure 1 we show the preliminary reduction of the CaII spectrum of the B2IV star $\alpha$ Pav ( $\mathrm{d}=57 \mathrm{pc}$ ) taken in November 1996 with the UHRF at the AAT. Four distinct absorption components are clearly seen, the weakest coincident with the projected velocity of the LIC component at $\mathrm{V}=-15.1$ $\mathrm{km} \mathrm{s}^{-1}$. A cloud component with a similar velocity has also been observed towards $\alpha$ Gru $(\mathrm{d}=18 \mathrm{pc})$ and $\epsilon$ Gru $(\mathrm{d}=23 \mathrm{pc})$, which are two stars lying within 10 degrees from the line-of-sight towards $\alpha$ Pav. One particularly interesting aspect of the velocity structure of this line-of-sight is the narrow absorption component seen at $-19.8 \mathrm{~km} \mathrm{~s}^{-1}$, whose b-value would indicate an upper limit to the temperature for this cloud of only $2100 \mathrm{~K}$. Note also the two broad components in the red wings of the photospheric profile which may be stellar line components, due to the binary nature of $\alpha$ Pav.

\section{Discussion}

Thus far, our program of UHRF observations of absorption in the LISM clearly shows that the velocity structure of diffuse cloudlets within $40 \mathrm{pc}$ is far from being simple. The absorption characteristics of the nearest cloud, the LIC, indicate that the Sun can only be partially embedded within it, and that at least in the direction of $\alpha$ Cen the LIC is either highly asymmetric or of very low column density. There seems to be a tendency for the LIC to be observed either on its own or not at all in lines-of-sight with high negative galactic latitudes (Crawford and Dunkin 1995). Thus far, the best interpretation of all the presently available CaII absorption data point to the fact that we are not observing classical cloud-like absorption features at all, but in fact we are detecting shocked filamentary sheet-like structures in the LISM. These "interstellar wisps" may well be remnants of the proposed supernova explosion that created the Local Bubble several million years ago.

The three key remaining problems to be answered about these LISM cloudlets are (1) how long can these wisps survive if they are embedded in the alleged hot million degree gas of the Local Bubble? (2) Are these wisps in pressure or ionization equilibrium, and (3) what happens at the interface between the $7000 \mathrm{~K}$ LIC gas and the hot LISM gas? Although our ground based UHRF data is providing important insights into the complex nature of the velocity structure of these local cloudlets, these three key questions concerning the LISM can only be answered by observations at other wavelengths, particularly using absorption spectroscopy in the ultraviolet regime, where a wide range of ionization states from many elements can be probed. Clearly the HST STIS instrument will play a key role in addressing many of these problems, and the upcoming FUSE satellite's observations of local OVI (1036 $\AA$ ) absorption will provide information on the relationship between both hot and cold local gas. Clearly much work has yet to be done in this field, and it is our hope to complete this UHRF survey within the next few years such that the kinematics of the LISM cloudlets can be well mapped and provide 
an important diagnostic database for understanding the sub-structure of the interstellar medium in regions of very low density and high ionization.

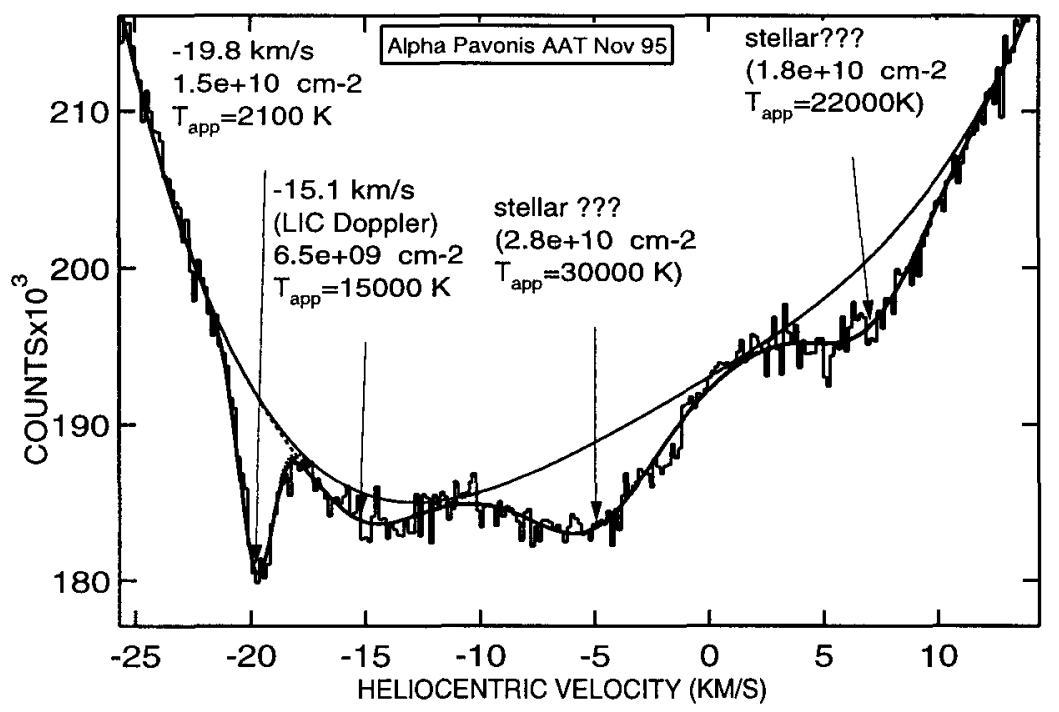

Fig. 1. UHRF fitted spectrum of the CaII absorption line towards Alpha Pav

\section{References}

Bertin, P., Lallement, R., Ferlet, R., and Vidal-Madjar, A. (1993): Astr. \& Astrophys., 278, 549-560

Crawford, I. (1994): The Observatory 114, 288-293

Crawford, I. and Dunkin, S. (1995): M.N.R.A.S., 273, 219-224

Crawford, I., Craig, N. and Welsh, B. (1997): Astr. \& Astrophys., 317, 889-897

Diego, F. et al. (1995) : M.N.R.A.S., 272, 323-332

Lallement, R. and Bertin, P. (1992): Astr. \& Astrophys., 266, 479-485

Lallement, R., Vidal-Madjar, A. and Ferlet, R. (1986): Astr. \& Astrophys., 168, 225-236

Linsky, J. and Wood, B. (1995): Astrophys. Journal, 463, 323-332

Vallerga, J., Vedder, P., Craig, N. and Welsh, B. (1993): Astrophys. Journal, 411, 729-749

Welsh, B., Craig, N., Vedder, P. and Vallerga, J. (1994): Astrophys. Journal, 437, 638-657 